\title{
Les applications de la microdissection laser en histologie
}

$>$ La microdissection laser permet d'isoler des cellules, phénotypiquement identiques, à partir d'une lame de microscope portant un tissu biologique, dans l'optique de réaliser des analyses moléculaires différentielles, spécifiques de ces populations isolées. La technologie s'applique notamment en oncologie, pour préciser des mécanismes moléculaires qui permettent d'adapter un traitement lié au diagnostic et à la recherche en biologie, mais aussi en criminalistique, pour la sélection tissulaire, en neurologie pour des études post-mortem sur des patients atteints de maladie d'Alzheimer, pour des études de clonalité à partir de cultures cellulaires, et en cytogénétique, pour décrypter les réarrangements chromosomiques. C'est le chaînon manquant entre observations cliniques et mécanismes physiologiques intrinsèques des tissus biologiques. Nous aborderons dans cette revue ses applications majeures. <

\section{La technologie laser}

Le rayonnement d'émission stimulé par la lumière laser (de l'anglais, light amplified stimulated emission radiation) est une source de lumière monochromatique et unidirectionnelle. Le laser a de nombreuses applications dans le domaine de la biologie et dans le domaine médical, et a permis la mise au point d'instruments de diagnostic et de traitements qui offrent de nombreuses applications [1]. Outre les approches diagnostiques et thérapeutiques classiques, un autre domaine de la technologie laser à l'échelle nanométrique contribue au développement de traitements ciblés, principalement dans le domaine du cancer, menant à la conception de stratégies nouvelles et innovantes pour l'administration de médicaments grâce à la chirurgie guidée par l'image. La mise au point de nanoparticules absorbant la lumière, non toxiques pour les tissus biologiques, peut produire des augmentations de température loca-

\section{Une voie ouverte aux études moléculaires}

\author{
Luc G. Legrès
}

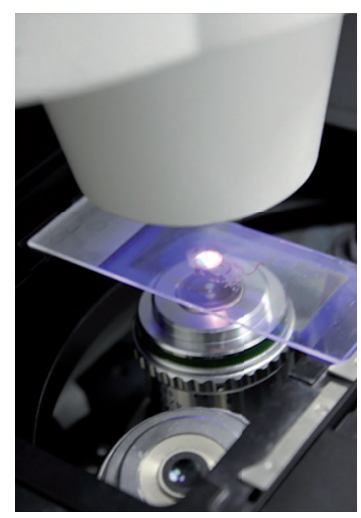

Institut de recherche Saint-Louis, Paris, France, UMR_S 976 Inserm, Université de Paris, Hôpital Saint-Louis, 1 avenue Claude-Vellefaux, F-75010 Paris, France. luc.legres@inserm.fr

lisées à l'échelle de la nanoparticule. Un laser à impulsions de haute énergie peut en effet déclencher un effet photothermique dans les tissus et provoquer des dommages cellulaires dans une zone tumorale lorsque la température dépasse un certain seuil [2].

Cette technologie est exploitée à l'extrême en jouant sur deux paramètres principaux, les impulsions laser courtes - à la femtoseconde - et l'énergie des faisceaux. Pour le diagnostic chez un patient, ou pour les études expérimentales, le tissu biologique peut être observé au microscope optique après un marquage soit immunohistochimique (par réaction enzymatique ou par l'excitation d'un ou plusieurs fluorophores après conjugaison avec un anticorps), soit histochimique (coloration standard ou spécifique de certains éléments cellulaires), soit encore directement, sur tissu broyé/lysé, pour une analyse moléculaire globale. La microdissection laser constitue un lien précieux entre ces approches (Figure 1). Développée en 1996, la microdissection au laser répond à la nécessité de miniaturiser les techniques analytiques applicables aux très petits nombres de cellules [3]. Dans les essais cliniques en oncologie, le profil moléculaire d'échantillons microdisséqués peut fournir des informations «omiques 》 globales qui, combinées à l'analyse morphologique des cellules, contribuent à mieux comprendre le diagnostic, de poser la base d'un pronostic et de donner de nouvelles informations sur les mécanismes cellulaires, les troubles génétiques, l'identification de biomarqueurs tumoraux pour une possible thérapie adaptée au patient [4].

\section{Les techniques de microdissection laser}

Quatre dispositifs de microdissection assistée par laser sont actuellement disponibles. Ils sont fondés sur différents principes de fonc- 


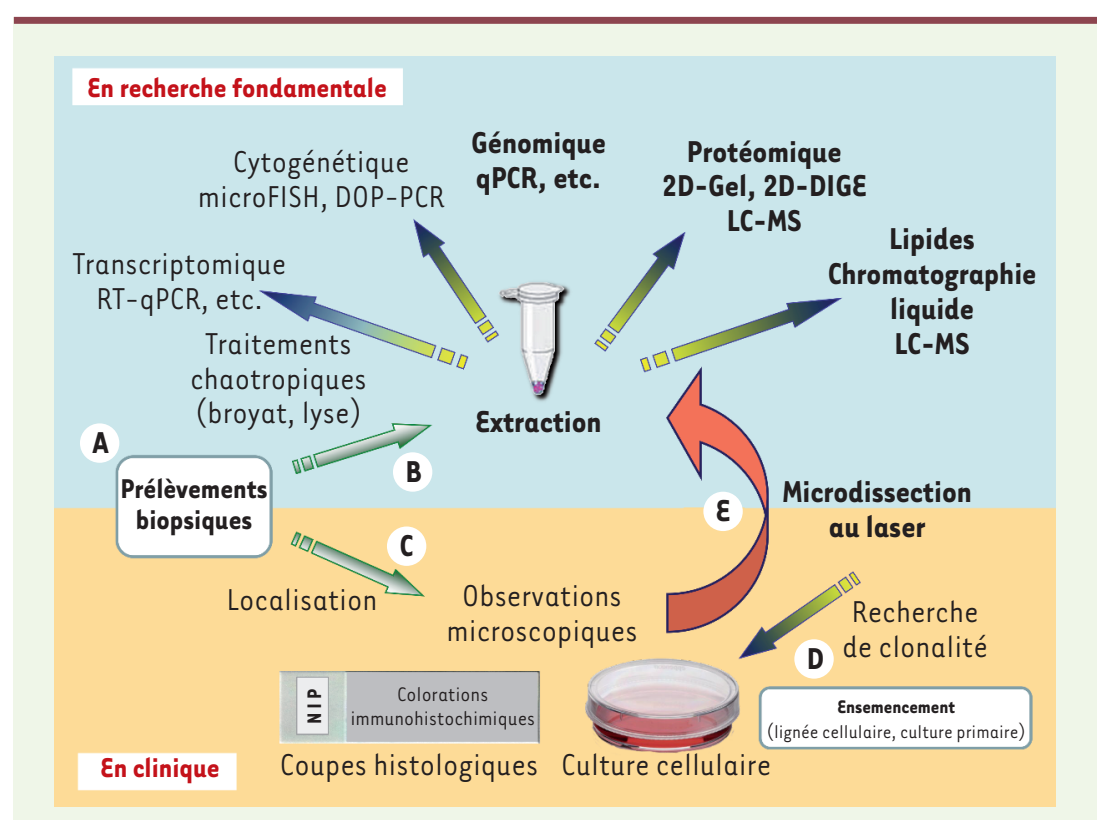

Figure 1. Intérêt de la microdissection laser pour la caractérisation d'échantillons en clinique et en recherche fondamentale. $A$. Prélèvement/biopsie de tissus biologiques. B. Broyat et/ou lyse des tissus prélevés pour analyses moléculaires sur tissus entiers. C. Localisation et observation histologique de coupes de tissus fixés puis inclus en paraffine (FFPE) ou congelés en azote liquide à - $196^{\circ} \mathrm{C}$. D. Mise en culture de cellules et observation au microscope photonique. $\varepsilon$. Étapes de microdissection au laser après sélections des cellules ou régions cellulaires d'intérêt pour des analyses moléculaires ciblées et recherche de clonalité par remise en culture de cellules isolées microdisséquées.

tionnement permettant d'extraire un tissu biologique d'une lame de microscope [5]. Les systèmes sont, d'une façon générale, composés d'un microscope (droit ou inversé) couplé à un faisceau laser (Iongueur d'onde supérieure à $750 \mathrm{~nm}$ dans la gamme infrarouge et/ou de $355 \mathrm{~nm}$ dans l'ultraviolet) avec des capacités de visualisation d'échantillons en fond clair, en fond noir et en fluorescence, et qui fonctionne sous le contrôle d'un programme informatique. Au grossissement approprié (le plus souvent à l'objectif x10, x20 ou x40), une ou plusieurs cellules isolées, voire des régions cellulaires, sont sélectionnées sur des coupes histologiques à l'aide d'un assistant graphique (dessin à main levée ou formes géométriques prédéfinies) disponible sur l'écran de l'ordinateur. L'épaisseur moyenne des coupes se situe entre 7 et $8 \mu \mathrm{m}$, d'une part afin de pouvoir correctement les regarder au microscope pour en distinguer les détails et, d'autre part, du fait que cette épaisseur représente un espace «de sécurité » car, si on observe bien la couche de cellules superficielles qui incite à les prélever, celle qui est en dessous (si l'épaisseur est trop grande) risque de fausser l'étude envisagée en collectant également des cellules non désirées. Certains travaux font état de prélèvements de 20 à $25 \mu \mathrm{m}$ d'épaisseur, mais l'objet de l'étude n'est pas le même, ni le tissu, qui doit alors être beaucoup plus homogène. Les cellules ou régions cellulaires sont sélectionnées/isolées puis collectées, selon l'appareil, soit dans un tube conventionnel (de type Eppendorf tube ${ }^{\circledR}$ ), soit sur un support spécifique (capsule ou tube à capuchon adhésif).

La largeur de coupe au laser est généralement inférieure à $1 \mu \mathrm{m}$ et le processus de microdissection laser ne modifie ni n'endommage la morphologie et la chimie de l'échantillon cible recueilli, ni les cellules environnantes. Pour cette raison, cette méthode est utile pour la collecte de cellules sélectionnées en vue d'analyses moléculaires portant sur l'étude de l'ADN, I'ARN ou de la/des protéine(s) d'intérêt [6].
Brièvement, les systèmes sont les suivants ${ }^{1}$ :

Le système Arcturus ${ }^{2}$. Initialement décrit en utilisant un laser infrarouge (IR), fait fondre une membrane plastique thermolabile portée par une capsule pour venir encoller le tissu biologique coloré sous-jacent qui a été préalablement sélectionné par un logiciel informatique. L'échantillon biologique microdisséqué est arraché comme par un système d'emporte-pièce lorsque la capsule est soulevée [3].

- De nos jours, au laser IR qui fusionne la membrane de la capsule au tissu sous-jacent, un laser ultra-violet (UV) a été ajouté pour découper le tissu et détruire sélectivement des cellules non pertinentes dans la zone d'intérêt.

Pour tout transfert de tissus, qualifié de «sans contact», effectué de la coupe histologique vers un support de récupération, il existe trois approches différentes :

- Celle du système PALM LMPC 3 où, à l'aide d'un microscope inversé, la région tissulaire sélectionnée est découpée par un laser UV puis catapultée sous un effet de pression, par défocalisation du laser et augmentation de son énergie, vers le creux d'un capuchon contenant la solution de récupération (un tampon d'extraction ou de lyse) [7].

- Celle du système LMD $^{4}$ où l'échantillon biologique, sélectionné puis découpé, tombe par gravité dans un

\footnotetext{
${ }^{1}$ Une description plus largement détaillée pourra être consultée dans la revue [5]. ${ }^{2}$ http://www.excilone.com/microdissecteur-arcturus-xt.html

${ }^{3}$ https://www.zeiss.fr/microscopie/produits/microdissection-laser/microbeam.html ${ }^{4}$ https://www.leica-microsystems.com/products/lightmicroscopes/details/product/leica-Imd7
} 
tube de récupération situé à l'aplomb de la lame histologique sur laquelle repose le tissu d'intérêt [8].

- Celle du système $\mu$ CellCut ${ }^{5}$ obtenue par un processus de coupe et de capture effectuées sur un capuchon recouvert d'un adhésif qui est positionné directement sur une membrane au revers de laquelle se trouve le tissu d'intérêt [9].

La microdissection laser, impliquant la visualisation directe et l'expertise d'un pathologiste, permet d'accéder à des populations de cellules définies ou à une région spécifique sur tout type d'échantillon de tissu, sélectionnant ainsi des populations de cellules phénotypiquement identiques. Cette technique ouvre la voie aux méthodes moléculaires dirigées vers des populations bien définies et constitue également un outil puissant pour les études portant sur un nombre limité de cellules, voire de cellule isolée (Figure 2).

\section{Applications en oncologie}

Les tissus biologiques réunissent différentes populations de cellules présentant des schémas de distribution particulièrement complexes, ce qui entraîne une hétérogénéité de leur composition cellulaire globale. Les tissus tumoraux contiennent également un large éventail de cellules tumorales disséminées dans un tissu conjonctif parfois abondant appelé stroma. Une approche différentielle des mécanismes moléculaires intrinsèques, dans des tissus biologiques impliquant principalement I'ADN, I'ARN et une ou plusieurs protéines, nécessite donc de s'affranchir de cette hétérogénéité par le prélèvement, et donc l'enrichissement, d'un certain type de cellules phénotypiquement identiques. Cette technologie remarquable a apporté de nouvelles connaissances sur I'ADN, notamment dans les recherches de perte d'hétérozygotie ( $\mathrm{LOH}$, de l'anglais loss of heterozygosity) [10], dans le cancer cutané après greffe rénale [11] $(\rightarrow)$ dans l'étude des profils d'expression des ARN [12], et, en particulier, dans les $\rightarrow$ Voir la nouvelle de L. Verneuil et A. Janin, $m / s n^{\circ} 3$, mars 2014, page 251 études de protéomique [13], en permettant d'identifier des marqueurs en tant que signatures moléculaires associées à un état pathologique donné. Cha et al. [14] ont identifié de tels marqueurs permettant de détecter une différenciation de cellules épithéliales normales du sein en cellules invasives malignes en combinant la microdissection laser et I'utilisation de techniques protéomiques dites bottom-up [15]. Ils ont pu montrer des variations dans la quantité des protéines, mais aussi des modifications importantes, en aval, touchant des facteurs de transcription, suggérant un rôle pour des voies de régulation géniques spécifiques dans la tumorigenèse du tissu mammaire [16].

Les méthodes d'analyses moléculaires les plus couramment utilisées pour étudier l'expression des gènes, telles que la RT-qPCR (quantitative reverse transcription polymerase chain reaction), une technique qui permet de réaliser une PCR quantitative à partir d'un échantillon d'ARN, fournissent des données pertinentes permettant de comparer entre eux plusieurs échantillons cellulaires phénotypiquement semblables. Cependant, il existe encore un grand nombre d'échantillons

${ }^{5}$ http://www.molecular-machines.com/solutions/molecular_pathology archivés pour lesquels des études moléculaires supplémentaires seraient souhaitables. Ainsi, les tissus biologiques préalablement fixés avec la formaldéhyde suivi d'une inclusion en paraffine (tissu dit FFPE, de l'anglais formaldehyde-fixed and paraffin-embedded) constituent une vaste bibliothèque d'échantillons pour la recherche biomédicale rétrospective. Pendant longtemps, l'extraction d'ARN, à partir des tissus FFPE, s'est avérée difficile en raison de la réticulation chimique ARN-protéine, due à l'étape de fixation dans la formaldéhyde qui a un impact important sur la quantité et la qualité de I'ARN pour l'analyse en aval [5]. Bien que la formaldéhyde reste le fixateur préféré des utilisateurs, d'autres solutions plus alcooliques contenant peu ou pas de formaldéhyde ont été commercialisées comme, par exemple, le fixateur RCL2 [5]. Aujourd'hui, grâce à l'émergence récente de nouvelles technologies moléculaires associées aux techniques de microdissection laser, telles que le séquençage de nouvelle génération (ou NGS, pour next-generation sequencing) ainsi que l'apparition de kits d'extraction adaptés à la récupération d'ARN à partir d'échantillons FFPE ou adaptés aux microquantités de tissus biologiques prélevés, il est possible de travailler sur des populations archivées afin de fournir des résultats beaucoup plus précis, reproductibles et significatifs par rapport à une pathologie donnée [16]. Landolt L et al. [17] ont montré, à partir de biopsies rénales issues d'archives, que l'ARN total pouvait être extrait en qualité et en quantité suffisante à partir d'échantillons FFPE pour permettre la préparation et le séquençage d'une bibliothèque d'ARN à l'aide de la technologue NGS et de kits d'extraction d'ARN disponibles dans le commerce.

\section{Analyse post-mortem du tissu cérébral}

Dans les maladies du cerveau, la zone touchée peut être de taille limitée et les événements pathologiques peuvent être liés à un type de cellule spécifique au sein d'un type de tissu qui est par ailleurs hétérogène. Dans le domaine des neurosciences, les études protéomiques de cellules neurales issues de populations cellulaires provenant de cerveaux post-mortem ont bénéficié des avancées technologiques de la microdissection laser afin d'isoler individuellement ces cellules, en association à la technique de chromatographie en phase liquide couplée à la spectrométrie de masse (LC/MS) [18]. Les méthodologies actuelles d'analyses moléculaires nécessitent une grande quantité de protéines, ce qui rend difficile les études spécifiques à un type de cellule. Des études antérieures hésitaient à utiliser du tissu FFPE pour la protéomique en raison d'inquiétudes concernant les 

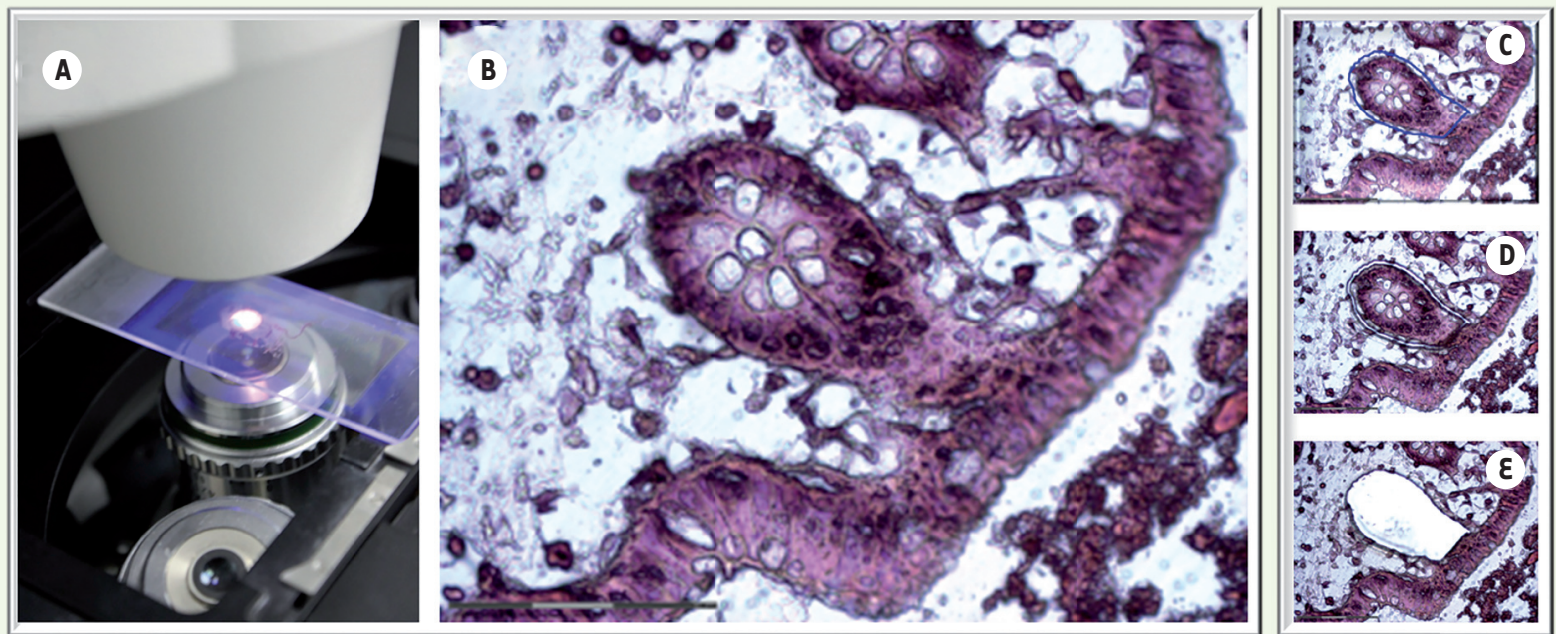

Figure 2. Les étapes de la dissection laser. A. Faisceau bleu du rayon laser UV. B. Observation histologique au microscope photonique (crypte colique). C. Sélection de la région d’intérêt (délimitée en bleu). $D$. Découpe au laser de la région sélectionnée. $\varepsilon$. Récupération de la région microdisséquée.

modifications induites par la formaldéhyde pouvant toucher et dégrader les protéines, au cours du traitement du tissu [19]. Cependant, bien que les tissus congelés instantanément (snap-freezed, en azote liquide à $-196^{\circ} \mathrm{C}$ ) soient toujours considérés comme la norme de référence pour les études de protéomique, il n'existe que très peu de blocs congelés de cerveaux humains provenant de patients post-mortem atteints de la maladie d'Alzheimer. Combinant la microdissection laser et la technique de spectrométrie de masse LC/MS, Drummond $\varepsilon S$ et al. [20] ont montré que des blocs de tissu FFPE stockés depuis 10 ans pouvaient être utilisés pour quantifier un nombre substantiel de protéines, si une technique d'extraction appropriée est utilisée.

\section{Recherche biologique juridique}

L'analyse ADN est fréquemment utilisée pour obtenir des informations à partir de matériel biologique afin de faciliter les enquêtes liées aux infractions pénales, à l'identification des victimes de catastrophes et aux enquêtes sur les personnes disparues [21] $(\rightarrow)$.

L'enquête sur une agression sexuelle implique souvent l'analyse des prélèvements vaginaux effectués

$\rightarrow$ Voir la Synthèse de F.X. Laurent et al., $m / s n^{\circ} 11$, novembre 2017, page 971

sur la victime à l'aide d'un écouvillon stérile. Si le sperme est présent, par hybridation à des sondes polymorphes de microsatellites, des traces d'ADN peuvent être décelées par Southern blot sur de multiples fragments d'ADN spécifiques d'un individu [21]. Cependant, dans les cas d'agresseurs azoospermiques (c'est-à-dire sans spermatozoïdes), bien que des tests de présomption puissent détecter la présence de sperme, aucun spermatozoïdes ne pouvaient être récupérés. Les prélèvements vaginaux peuvent ainsi ne pas fournir de profil ADN permettant d'effectuer une recherche dans une base de données suspecte, car le signal des cellules présentes (cellules épithéliales et leucocytes provenant du canal éjaculatoire et de l'urètre) serait masqué par celui du très grand nombre de cellules femelles recueillies [22].

Les échantillons d'agression sexuelle sont parmi les plus fréquemment analysés dans un laboratoire médico-légal et une grande partie de ces cas restent non résolus.

Les spermatozoïdes sont traditionnellement séparés des cellules épithéliales vaginales par extraction différentielle. Les cellules épithéliales sont en effet préférentiellement lysées par traitement dans un mélange de détergeant (SDS, dodécylsulfate de sodium) et de protéinase $K$, tandis que les spermatozoïdes y sont résistants et peuvent donc ensuite être séparés des débris cellulaires par centrifugation [21]. Elliot et al. ont montré que la microdissection laser surpasse l'extraction différentielle pour la récupération de I'ADN des spermatozoïdes au cours d'une enquête médico-légale sur le viol et d'autres agressions sexuelles, en particulier dans les cas où seuls quelques spermatozoïdes sont présents, mélangés à de plus grande quantité de cellules vaginales [24]. D'autres études ont utilisé la microdissection laser pour isoler les spermatozoïdes [25] et les cellules des follicules pileux [26], ainsi que pour faciliter le test de paternité sur des tissus provenant de tissu abortif [27]. De récentes technologies de génotypage permettent d'obtenir un profil génétique fiable pour l'identification d'un suspect. Le défi ultime se pose lorsque des profils génétiques doivent être extraits d'un mélange composé de matériel biologique provenant de deux personnes ou plus [28]. 
Au microscope, différents types de cellules peuvent être distingués en fonction de leurs caractéristiques morphologiques, de diverses techniques de marquage chimique ou de marquage par fluorescence. Ainsi, des méthodes fondées sur l'hybridation de fluorescence in situ (ou FISH, fluorescence in situ hybridization) ont été combinées à la microdissection laser pour identifier et isoler simultanément des cellules mâles (spermatozoïdes, cellules épithéliales et leucocytes) [29]. Plusieurs laboratoires ont rapporté avoir utilisé le kit SPERM HY-LITER TM [30], qui contient un anticorps monoclonal de souris spécifique des têtes de spermatozoïde humain, donc de l'acrosome, et n'a pas de réaction croisée avec d'autres fluides corporels. II est reconnu par un anticorps secondaire conjugué à un fluorochrome (Alexa Fluor ${ }^{\circledR} 488$ ) pour marquer spécifiquement les spermatozoïdes humains avant leur capture par microdissection laser [31]. Un deuxième fluorochrome (le DAPI ou 4',6-diamidino-2-phénylindole), qui se lie fortement à l'ADN et marque les noyaux de toutes les cellules présentes dans l'échantillon, est incorporé dans le kit permettant, avec un filtre double, d'examiner les échantillons dans lesquels tous les noyaux apparaissent en bleu alors que les têtes de spermatozoïdes apparaissent en vert (dans ces conditions, les têtes de spermatozoïdes marquées au DAPI sont masquées par la lumière verte du fluorochrome Alexa). Certains anticorps monoclonaux utilisés peuvent se lier à plus d'une de protéine de sperme, indiquant que le même déterminant antigénique est fréquemment partagé par plus d'une protéine de sperme [32] (sans qu'il soit précisé lesquelles pour des raisons de protection d'un brevet industriel). La coloration standard hématoxyline/éosine (ou $H E)^{6}$ des échantillons n'est pas aussi efficace car elle n'offre pas le même niveau d'identification des spermatozoïdes qui ne peuvent être trouvés que lorsque la proportion de sperme par rapport aux cellules épithéliales est supérieure à $20 \%$. Le kit SPERM HY-LITER TM permet quant à lui de facilement détecter les spermatozoïdes à des concentrations inférieures à $10 \%$ dans le sperme [28].

En criminalistique, des frottis fraîchement préparés ou archivés peuvent être utilisés, par exemple, pour l'isolement de spermatozoïdes ou de cellules épithéliales. Même des années après un crime, une empreinte génétique à partir de cellules spécifiques peut aider à identifier le coupable [33]. Un logiciel de traitement et d'analyse d'images, l'AxioVision Commander Script, associé au système PALM MicroBeam, peut être optimisé pour détecter et identifier automatiquement des cellules. Vandewoestyne M. et al. ont démontré que cette technologie avait une sensibilité suffisante pour la détection de spermatozoïdes colorés au moyen du kit SPERM HY-LITER TM [34].

\section{Manipulation de cellules vivantes}

La plupart des applications de micromanipulation au laser se concentrent sur l'isolement de cellules spécifiques provenant de différents types de tissus fixés ou congelés. En regard de critères morpholo-

${ }^{6}$ Coloration bichromatique qui se compose d'un colorant nucléaire, l'hématoxyline, et d'un colorant cytoplasmique, l'éosine. giques, de comportement de croissance, de marqueurs de surface définis ou d'expression génique visualisée par la présence d'un gène rapporteur tel que celui codant la GFP (green fluorescent protein), la microdissection par laser ultraviolet pulsé permet l'isolement et la remise en culture de cellules vivantes à partir de cultures de cellules adhérentes [35].

À cette fin, de nouveaux protocoles et équipements spéciaux, propres à chaque système, ont été développés pour les collecter, notamment concernant le confinement des cellules en milieu stérile, de façon similaire à celui d'une étuve (température maintenue à $37{ }^{\circ} \mathrm{C}$ et apport de $5 \%$ en $\mathrm{CO}_{2}$ ) mais en ôtant le milieu pour ne laisser qu'une couche humide.

Avec le système Arcturus, les cellules sont cultivées sur une lame recouverte d'une membrane de PEN (polyéthylène naphthalate) sur laquelle est déposée une couche de poly-L-lysine pour renforcer l'adhérence des cellules et, éventuellement, de laminine, utilisée aussi pour l'amélioration de l'adhérence cellulaire mais qui favorise, en plus, la migration et la prolifération de cellules de divers types, en particuliers les cellules épithéliales, les myocytes et les myoblastes $(\rightarrow)$.

La lame est placée dans une boîte de culture cellulaire. Les agencements de la lame de membrane PEN et de la capsule de recueil (capture) dans l'instrument favorisent une découpe au laser UV et une capture au laser $I R$ des zones marquées qui sont ainsi collectées [37].

Des recherches antérieures ont déjà décrit l'utilisation de la

$\rightarrow$ Voir la Mini-synthèse de P. Rousselle, $m / s$ $n^{\circ} 10$, octobre 2002 , page 989 microdissection laser par catapultage sous pression (LMPC, laser microdissection and pression catapulting) pour l'isolement de cellules vivantes [38]. Pour effectuer la collecte des cellules souhaitées, le milieu de culture est retiré, laissant uniquement une couche résiduelle humide au-dessus des cellules. Les cellules sont cultivées dans une boite de Pétri dont le fond en plastique est remplacé par une membrane de téflon seulement perméable au gaz et recouverte d'une membrane de PEN sur laquelle croissent les cellules. Les paramètres d'énergie et de focalisation laser dépendent des caractéristiques de l'échantillon (taille, épaisseur, humidité) et de l'objectif appliqué. L'énergie doit être augmentée pour l'éjection de matières humides, plus lourdes que les matières sèches [39].

Le principe de la microdissection laser avec transfert gravitaire peut aussi s'appliquer à la capture de cellules vivantes [40]. Des cellules, ou une région d'intérêt comportant des cellules vivantes, est découpée dans une boîte de Pétri montée sans couvercle sur un microscope droit. Les cellules, après découpe, 
tombent alors dans une autre boîte de Pétri emplie de milieu de culture et placée directement sous l'échantillon. Cette méthode a permis de montrer que $70 \%$ de cellules HeLa ainsi isolées pouvaient donner lieu à des colonies clonales. Le principal avantage de cette méthode par rapport à la LMPC est la collecte directe de l'échantillon dans une autre boîte de culture. L'identification de mécanismes d'interaction entre des cellules de mélanome humain et leur microenvironnement a ainsi été entreprise par microdissection laser suivi d'une analyse par spectrométrie de masse : à partir de cultures primaires issues de mélanome et métastatiques provenant de cultures de peau [4l]; à partir de culture de la lignée cellulaire HepG2 de carcinome hépatocellulaire humain [42]; ou pour une analyse d'un adénocarcinome pancréatique [43].

La chambre MMI LiveCell est constituée, pour la culture initiale de cellules, d'une boîte de culture cellulaire dans laquelle se trouve un anneau métallique avec une membrane contenant les cellules ensemencées, et une chambre de microdissection à fond en silicone perméable aux UV [44]. La plupart des lignées de cellules souches sont actuellement cultivées à des densités élevées sur des couches nourricières de fibroblastes de souris. L'isolement de cellules pluripotentes spécifiques des fibroblastes environnants et des cellules de différenciation doit être rapide et précis. Avec cette méthode, un équilibre parfait entre vitesse et précision permet d'isoler et de remettre en culture les cellules souches cibles, sans aucun effet secondaire, tels que des modifications du caryotype.

\section{Applications en cytogénétique}

La cytogénétique est une branche de la génétique qui s’intéresse au lien entre les chromosomes et le comportement des cellules. McClintock et Creighton ont initialement démontré que la recombinaison cytologique de chromosomes marqués était rapportée à la recombinaison de gènes [45]. Ainsi, par exemple, Janet Rowley a observé que le chromosome Philadelphie était le résultat d'une translocation anormale des chromosomes 9 et 22, résultant d'une fusion appelée BCR-ABLl, dont l'identification permet de diagnostiquer la leucémie myéloïde chronique (LMC) [46]. Les progrès réalisés en cytogénétique moléculaire ont conduit à l'utilisation de sondes fluorescentes (technologie FISH) pouvant s'hybrider in situ sur des préparations chromosomiques [47]. Ce changement a considérablement augmenté l'utilisation des techniques de marquage car les sondes marquées par fluorescence se révèlent plus précises. D’autres progrès dans la micromanipulation et l'examen des chromosomes ont conduit à la technique de la microdissection des chromosomes grâce à laquelle des aberrations de structure pourraient être isolées, clonées et étudiées de manière plus détaillée. La dissection de chromosomes a été initialement développée à l'aide d'un micromanipulateur dans le cadre d'études entreprises sur des chromosomes polytènes de Drosophila melanogaster afin d'obtenir des marqueurs $A D N$ de régions chromosomiques spécifiques [48]. Après l'avènement de la technique de marquage des chromosomes au Giemsa (mélange de deux colorants rose violacé, le bleu de méthylène et l'éosine) qui facilite leur identification et de la PCR, la technique de microdissection des chromosomes a été largement utilisée dans la recherche en génomique [49]. Le développement ultérieur de la technologie FISH et la mise en place de DOP-PCR (degenerate oligonucleotide primed-PCR) [50] ont permis de générer des sondes chromosomiques par microdissection (sous un grossissement $\times 100$ à immersion dans l'huile) qui est appliquée lorsque l'identification du chromosome par la coloration à la trypsine-Giemsa est compliquée ou impossible en raison de la morphologie chromosomique [51]. De plus en plus de sites chromosomiques dans le génome humain ont été identifiés en tant que lésions primaires dans des maladies génétiques spécifiques ou des cancers, comme dans la maladie de Huntington [52]. L'identification de séquences de régions chromosomiques humaines associées à des maladies génétiques ou à des cancers spécifiques constitue l'approche la plus répandue dans les dernières recherches cliniques pour comprendre l'implication de gènes liés à la maladie.

\section{Conclusion}

De nombreuses avancées technologiques, en particulier dans le domaine de l'imagerie, ont rendu possible l'observation et la collecte d'échantillons biologiques solides chez l'homme et les biopsies peuvent être analysées au microscope après immunohistochimie ou conservées pour des analyses moléculaires ultérieures. La caractérisation génétique de l'hétérogénéité cellulaire observée dans les tissus est essentielle pour comprendre les événements moléculaires survenant dans les processus physiopathologiques. La technique de la microdissection laser revêt une grande importance dans ce domaine pour des analyses moléculaires au niveau de la cellule. Cela a contribué à apporter une plus grande précision dans la sélection des tissus biologiques visibles au microscope en fonction de leur état physiopathologique ou non. Cette précision dans la récupération des échantillons biologiques est principalement fonction de l'expertise dans la reconnaissance des tissus biologiques. La possibilité d'utiliser différents marqueurs en tant que colorants spécifiques ou non, d'anticorps conjugués ou non, a considérablement élargi les possibilités de recherche future à partir de tissus congelés ou même inclus en paraffine après fixation. Cependant, s'affranchir de la sélection manuelle faite par le pathologiste après une observation au microscope d'un tissu est un défi qui attire un certain nombre de chercheurs qui envisagent une approche 
«haut-débit» de la microdissection laser. La microdissection d'expression (xMD), par exemple, est une méthode d'isolement cellulaire ou subcellulaire indépendante de l'opérateur qui, pour des analyses haut-débit en aval, facilite une sélection et une capture de cellules [53]. Cette technologie ne s'appliquant qu'au système Arcturus, utilise le chromogène 3,3-diaminobenzidine (DAB) à base de peroxydase pour absorber l'énergie laser infrarouge, ce qui entraîne le chauffage focal d'un film polymère d'éthylène acétate de vinyle (EVA) en contact avec les cellules colorées. Les résultats obtenus indiquent que différents types de cellules ont pu être prélevés par cette méthode, et qu'il existe des différences de qualité de I'ARN contrairement à la microdissection classique [54]. Une autre approche technologique envisagée par Brasko et al. consiste en l'isolement de cellules par microscopie assistée par ordinateur qui combine des algorithmes d'analyse d'images, l'apprentissage automatique et la microscopie à haut débit pour reconnaître les cellules individuelles et guider automatiquement l'extraction par microdissection laser [55]. Malgré l'obtention de certains résultats, cette approche est limitée par plusieurs contraintes, telles que la configuration du microscope (configuration des paramètres du microscope, recherche du focus et sélection de la région et des cellules d'intérêt) qui revêt une importance primordiale. De plus, des phénotypes rares nécessitent une recherche dans plus d'images pour trouver les cellules intéressantes à isoler. Malgré les capacités de la technologie à cibler, à isoler et à analyser des cellules isolées, car elle repose sur un ensemble diversifié de technologies complexes, il existe plusieurs sources d'erreurs potentielles.

Toute méthode de préparation des tissus doit être un compromis entre l'obtention d'un bon contraste morphologique et la possibilité de conserver le matériel biologique pour des analyses moléculaires ultérieures et il semble encore bien difficile de s'affranchir de l'expertise et des connaissances du médecin pathologiste pour discerner des types histologiques avec le moins d'erreurs possibles [5]. De fait, il est important de noter que la microdissection tissulaire ne peut pas encore être mise en œuvre dans le contexte du diagnostic quotidien car elle nécessite des mises au point techniques spécifiques qui dépendent des tissus (texture, fixation, inclusion, congélation, conditions de préservation de l'intégrité du matériel biologique) et qui demande du temps pour sa mise en place, par des ajustements techniques, mais elle convient parfaitement aux protocoles de recherche définis dans le cadre d'un partenariat multiple. Un support méthodologique à long terme est nécessaire à la fois pour la maintenance technique du système et pour les protocoles développés par les chercheurs, les techniciens, les ingénieurs et les étudiants.

\section{Perspectives}

Dans PubMed, le principal moteur de recherche de données bibliographiques, sous les mots clés «laser microdissection», nous avons observé depuis 1996 un nombre toujours croissant de publications (Figure 3).

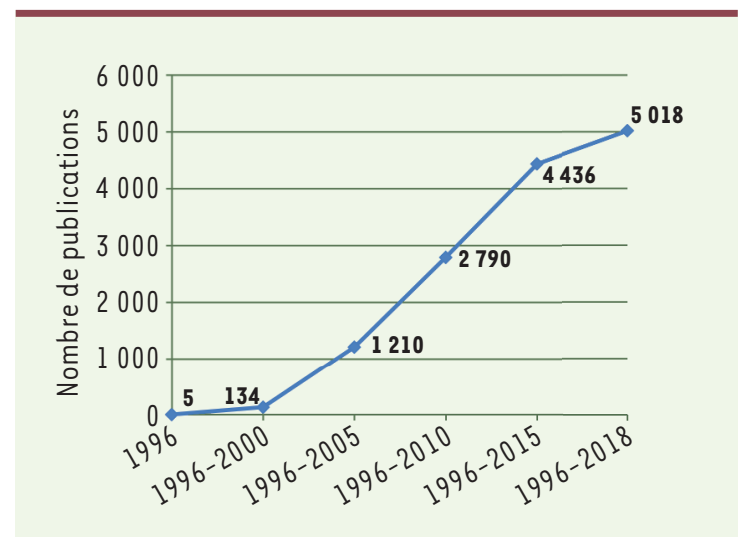

Figure 3. Recherche bibliographique dans la base de données PubMed avec «laser microdissection 》 comme mot clé en fonction des années.

Aujourd'hui, nous devons réfléchir à chaque étape en amont et en aval de l'étape de microdissection. Il est fondamental de comprendre que l'approche technique dépendra essentiellement du tissu étudié, de sa texture et en particulier de la présence ou de l'absence de nucléases ou de protéases, plus abondantes dans le pancréas, la rate ou même les poumons. En réalité, l'objectif est de développer une méthodologie innovante pour améliorer le diagnostic des tumeurs et la gestion des patients, méthodologie qui, dans de très nombreux cas, doit reconnaître la microdissection laser comme une technique de premier plan.

Dans un proche avenir, un nombre croissant de laboratoires de recherche devraient développer des plates-formes de haut niveau pour de telles études portant sur des essais cliniques pratiqués sur des patients. À terme, la validation de cette technique permettrait des approches thérapeutiques individualisées et donc une meilleure gestion de la qualité de soin des patients.

En raison de la spécificité et de la grande efficacité acquise lors de son utilisation en science médicale, la technique est devenue un outil puissant pour également répondre à un certain nombre de questions dans divers domaines de la biologie végétale incluant l'analyse comparative du transcriptome de différents tissus tels que les parties reproductrices, les méristèmes, les organes latéraux ou les racines [56]. Les protocoles envisagés sont spécifiquement adaptés à la microdissection de cellules et de tissus végétaux, dont les propriétés (notamment les parois cellulaires cellulosiques et les grandes vacuoles hydrolytiques) présentent des défis uniques pour la technologie. $\diamond$ 


\section{SUMMARY}

Laser microdissection applications in histology: an open way to molecular studies

One of the most fascinating aspects of the use of a laser beam in the field of biology has emerged with the development of devices able to perform fine dissections of biological tissues. Laser microdissection can collect phenotypically identical cells from tissue regions laid on a microscope slide in order to make differential molecular analyses on these microdissected cells. Laser microdissection can be used many areas including oncology to specify molecular mechanisms that enable to adapt a treatment related to diagnosis and research in biology, but also forensic science for tissue selection, neurology for post-mortem studies on patients with Alzheimer's disease, for clonality studies from cell cultures and cytogenetics to decipher chromosomal rearrangements. This technology represents the missing link between clinical observations and the intrinsic physiological mechanisms of biological tissues and its major applications will be addressed here. $\diamond$

\section{REMERCIEMENTS}

L'auteur tient à remercier le Pr Philippe Bertheau, PUPH, Hôpital Saint-Louis, Service de Pathologie (IRSL, Université de Paris, ex-Université Paris-Diderot) pour sa lecture attentive et les critiques judicieuses du manuscrit et le Dr Thierry Jouault, Inserm UMR_S 995, Lille, pour la finalisation de sa mise en forme.

\section{LIENS D'INTÉRÊT}

L'auteur déclare n'avoir aucun lien d'intérêt concernant les données publiées dans cet article.

\section{RéFÉRENCES}

1. Legres L, Chamot, C., Varna, M., Janin, A. The laser technology: new trends in biology and medicine. J Modern Physics $2014: 26779$.

2. Avedisian CT, Cavicchi RE, McEuen PL, Zhou X. Nanoparticles for cancer treatment: role of heat transfer. Ann NY Acad Sci $2009 ; 1161: 62-73$.

3. Emmert-Buck MR, Bonner RF, Smith PD, et al. Laser capture microdissection. Science 1996 ; 274 : 998-1001.

4. Domazet B, Maclennan GT, Lopez-Beltran A, et al. Laser capture microdissection in the genomic and proteomic era: targeting the genetic basis of cancer. Int J Clin Exp Pathol 2008 ; $1:$ : 475-88.

5. Legres LG, Janin A, Masselon C, Bertheau P. Beyond laser microdissection technology: follow the yellow brick road for cancer research. Am J Cancer Res $2014 ; 4: 1-28$.

6. Espina V, Wulfkuhle JD, Calvert VS, et al. Laser-capture microdissection. Nat Protoc 2006 ; $1: 586-$ 603.

7. Bohm M, Wieland I, Schutze K, Rubben H. Microbeam MOMeNT: non-contact laser microdissection of membrane-mounted native tissue. Am J Pathol 1997 ; $151: 63-7$.

8. Kolble K. The LEICA microdissection system: design and applications. J Mol Med (Berl) $2000 ; 78$ : B24-5.

9. Böhm, MSC, Wieland I LN. Membrane-based laser microdissection in molecular oncology. Onkologie 1999: 296-301.

10. Bertheau P, Plassa LF, Lerebours F, et al. Allelic loss detection in inflammatory breast cancer: improvement with laser microdissection. Lab lnvest 2001 ; 81 : 1397-402.

11. Verneuil L, Janin A. Cancer cutané après transplantation rénale : contribution des cellules épithéliales du rein greffé. Med Sci (Paris) 2014 ; $30: 251-2$.

12. Rosenberg AZ, Armani MD, Fetsch PA, et al. High-throughput microdissection for next-generation sequencing. PLoS One 2016; 11 : e0151775.

13. De Marchi T, Braakman RB, Stingl C, et al. The advantage of laser-capture microdissection over whole tissue analysis in proteomic profiling studies. Proteomics $2016 ; 16: 1474-85$.

14. Cha S, Imielinski MB, Rejtar T, et al. In situ proteomic analysis of human breast cancer epithelial cells using laser capture microdissection: annotation by protein set enrichment analysis and gene ontology. Mol Cell Proteomics $2010 ; 9$ : 2529-44.

15. Zhang Y, Fonslow BR, Shan B, et al. Protein analysis by shotgun/bottom-up proteomics. Chem Rev $2013 ; 113: 2343-94$.
16. Magel L, Bartels S, Lehmann U. Next-generation sequencing analysis of laser-microdissected formalin-fixed and paraffin-embedded (FFPE) tissue specimens. Methods Mol Biol 2018 ; 1723 : 111-8.

17. Landolt L, Marti HP, Beisland C, et al. RNA extraction for RNA sequencing of archival renal tissues. Scand J Clin Lab Invest 2016 ; 76 : 426-34.

18. Hondius DC, Hoozemans JJM, Rozemuller AJM, et al. A Laser microdissectionliquid chromatography-tandem mass spectrometry workflow for postmortem analysis of brain tissue. Methods Mol Biol 2018 ; 1723 : 371-83.

19. Rekhter MD, Chen J. Molecular analysis of complex tissues is facilitated by laser capture microdissection: critical role of upstream tissue processing. Cell Biochem Biophys $2001 ; 35: 103-13$.

20. Drummond ES, Nayak S, Ueberheide B, Wisniewski T. Proteomic analysis of neurons microdissected from formalin-fixed, paraffin-embedded Alzheimer's disease brain tissue. Sci Rep $2015 ; 5$ : 15456.

21. Laurent FX, Vibrac G, Rubio A, et al. Les nouvelles technologies d'analyses ADN au service des enquêtes judiciaires. Med Sci (Paris) 2017 ; 33 : 971-8.

22. Gill P, Jeffreys AJ, Werrett DJ. Forensic application of DNA fingerprints. Nature 1985 ; $318: 577-9$.

23. Murray C, McAlister C, Elliott K. Identification and isolation of male cells using fluorescence in situ hybridisation and laser microdissection, for use in the investigation of sexual assault. Forensic Sci Int Genet $2007 ; 1: 247-52$.

24. Elliott K, Hill DS, Lambert C, et al. Use of laser microdissection greatly improves the recovery of DNA from sperm on microscope slides. Forensic Sci Int $2003 ; 137: 28-36$.

25. Di Martino D, Giuffre G, Staiti N, et al. Single sperm cell isolation by laser microdissection. Forensic Sci Int 2004 ; 146 (suppl) : S151-3.

26. Di Martino D, Giuffre G, Staiti N, et al. Laser microdissection and DNA typing of cells from single hair follicles. Forensic Sci Int 2004; 146 (suppl) : S155-7.

27. Bauer M, Thalheimer A, Patzelt D. Paternity testing after pregnancy termination using laser microdissection of chorionic villi. Int J Legal Med $2002 ; 116: 39-42$.

28. Costa S, Correia-de-Sa P, Porto MJ, Caine L. The use of laser microdissection in forensic sexual assault casework: Pros and cons compared to standard methods. J Forensic Sci 2017 ; 62 : 998-1006.

29. Anslinger K, Bayer B, Mack B, Eisenmenger W. Sex-specific fluorescent labelling of cells for laser microdissection and DNA profiling. Int J Legal Med 2007 ; $121: 54-6$.

30. Miller KW, Old J, Fischer BR, et al. Developmental validation of the SPERM HY-LITERTM kit for the identification of human spermatozoa in forensic samples.J Forensic Sci $2011 ; 56: 853-65$.

31. Vandewoestyne M, Van Hoofstat D, Van Nieuwerburgh F, Deforce D. Suspension fluorescence in situ hybridization (S-FISH) combined with automatic detection and laser microdissection for STR profiling of male cells in male/female mixtures. Int J Legal Med 2009 ; 123 : 441-7.

32. Lee Cy, Wong $\varepsilon$, Richter DE, Menge AC. Monoclonal antibodies to human sperm antigens--II. J Reprod Immunol $1984 ; 6: 227-38$.

33. Burgemeister R. New aspects of laser microdissection in research and routine.J Histochem Cytochem 2005 ; 53 : 409-12.

34. Vandewoestyne M, Van Hoofstat D, Van Nieuwerburgh F, Deforce D. Automatic detection of spermatozoa for laser capture microdissection. Int J Legal Med 2009 ; 123 : 169-75.

35. Vogel A, Horneffer V, Lorenz $K$, et al. Principles of laser microdissection and catapulting of histologic specimens and live cells. Methods Cell Biol 2007 ; $82: 153-205$.

36. Rousselle P. Laminine 5, migration cellulaire et cancer. Med Sci (Paris) $2002 ; 18: 989-94$.

37. Bouchard R, Chong T, Pugazhenthi S. Laser capture microdissection of neurons from differentiated human neuroprogenitor cells in culture. J Vis Exp 2013 : e50487.

38. Stich M, Thalhammer S, Burgemeister R, et al. Live cell catapulting and recultivation. Pathol Res Pract 2003 ; 199 : 405-9.

39. Terstegge S, Rath BH, Laufenberg I, et al. Laser-assisted selection and passaging of human pluripotent stem cell colonies. J Biotechnol 2009 ; 143 : 224-30.

40. Podgorny OV. Live cell isolation by laser microdissection with gravity transfer. J Biomed Opt 2013; $18: 55002$.

41. Hood BL, Grahovac J, Flint MS, et al. Proteomic analysis of laser microdissected melanoma cells from skin organ cultures. J Proteome Res $2010 ; 9: 3656-63$.

42. Mustafa A, Cenayko C, Mitry RR, Quaglia A. Laser microdissection microscopy: application to cell culture. Methods Mol Biol $2012 ; 806$ : 385-92. 


\section{RÉFÉRENCES}

43. Funel $N$, Giovannetti $\varepsilon$, Del Chiaro $M$, et al. Laser microdissection and primary cell cultures improve pharmacogenetic analysis in pancreatic adenocarcinoma. Lab Invest 2008 ; 88 : 773-84.

44. Gousset K, Gordon A, Kumar Kannan S, Tovar J. A novel microproteomic approach using laser capture microdissection to study cellular protrusions. Int J Mol Sci 2019;20:1172.

45. Creighton HB, McClintock B. A Correlation of cytological and genetical crossing-over in zea mays. Proc Natl Acad Sci USA $1931 ; 17: 492-7$.

46. Rowley JD. Letter: A new consistent chromosomal abnormality in chronic myelogenous leukaemia identified by quinacrine fluorescence and Giemsa staining. Nature $1973 ; 243: 290-3$.

47. Amann R, Fuchs BM. Single-cell identification in microbial communities by improved fluorescence in situ hybridization techniques. Nat Rev Microbiol 2008 ; $6: 339-48$.

48. Scalenghe F, Turco $\varepsilon$, Edström JE, et al. Microdissection and cloning of DNA from a specific region of Drosophila melanogaster polytene chromosomes. Chromosoma $1981 ; 82$ : 205-16.

49. Lüdecke HJ, Senger G, Claussen U, Horsthemke B. Cloning defined regions of the human genome by microdissection of banded chromosomes and enzymatic amplification. Nature $1989 ; 338: 348$ 50 .

50. Telenius H, Carter NP, Bebb CE, et al. Degenerate oligonucleotide-primed PCR: general amplification of target DNA by a single degenerate primer. Genomics $1992 ; 13: 718-25$

51. Deng HX, Yoshiura K, Dirks RW, et al. Chromosome-band-specific painting: chromosome in situ suppression hybridization using PCR products from a microdissected chromosome band as a probe pool. Hum Genet $1992 ; 89: 13-7$.
52. Magenis RE, Gusella J, Weliky K, et al. Huntington disease-linked restriction fragment length polymorphism localized within band pl6.l of chromosome 4 by in situ hybridization. Am J Hum Genet 1986 ; $39: 383-91$.

53. Tangrea MA, Chuaqui RF, Gillespie JW, et al. Expression microdissection: operator-independent retrieval of cells for molecular profiling. Diagn Mol Pathol 2004 ; 13 : 207-12.

54. Kumar B, Rosenberg AZ, Choi SM, et al. Cell-type specific expression of oncogenic and tumor suppressive microRNAs in the human prostate and prostate cancer. Sci Rep 2018; $8: 7189$.

55. Brasko C, Smith K, Molnar C, et al. Intelligent image-based in situ single-cell isolation. Nat Commun $2018 ; 9: 226$.

56. Gautam V, Sarkar AK. Laser assisted microdissection, an efficient technique to understand tissue specific gene expression patterns and functional genomics in plants. Mol Biotechnol $2015 ; 57: 299-308$.

TIRÉS À PART

L.G. Legrès

\title{
Du cerveau aux neurosciences : Itinéraires dans la longue durée
}

ze journée d'étude du Comité pour l'histoire de l'Inserm

\section{2 janvier 2020 de $9 \mathrm{~h}$ à $18 \mathrm{~h}$}

\author{
Amphithéâtre Michelet - Sorbonne \\ 46 rue Saint Jacques - Paris $5^{e}$
}

Les neurosciences occupent une place croissante au sein de la recherche médicale et scientifique. Plus qu'une discipline, elles articulent un ensemble de champs liés à la connaissance du cerveau et du système nerveux et interrogent les rapports complexes de l'homme à son environnement et à ses semblables. De la recherche biomédicale aux sciences cognitives, acteurs, démarches et organisations qui structurent les neurosciences sont diverses. Souvent convergentes, elles peuvent également s'avérer concurrentes. Alors que les découvertes et les défis des neurosciences sont relayés par les médias, ce domaine entre espoir et inquiétude suscite l'intérêt croissant du grand public. Au prisme des dernières techniques de l'imagerie médicale, le cerveau fascine. De manière désordonnées et, parfois, mal informées, les « neurosciences » semble partout... Intégrées, parfois sans discernement, dans nombre de débats, elles sont mobilisées à propos des enjeux de société et de santé publique, d'éducation, de vieillissement et nourrissent chez certains les espoirs d'un homme « augmenté ». Un regard plus réfléchi ne serait-il pas dès lors nécessaire?

C'est par l'histoire que la troisième journée d'étude du Comité pour l'histoire de l'Inserm espère favoriser une réflexion plus sereine. Elle abordera tout d'abord l'évolution de la connaissance, de l'exploration et des représentations du cerveau du $x v l^{e}$ siècle aux dernières innovations du xxl ${ }^{e}$ siècle. La construction historique de la définition de la maladie et de l'élaboration des thérapeutiques articulée à la mise en place d'un encadrement institutionnel et hospitalier, sera ensuite interrogée, en posant la question de la place et de la représentation des patients au sein du système de santé, et plus largement de la société.

Ce rendez-vous constituera un nouveau temps de rencontre et d'échanges entre historiens, chercheurs en sciences humaines et sociales, témoins, scientifiques et acteurs institutionnels et associatifs. En tournant leur regard vers le passé, ils auront à l'esprit les problématiques actuelles ... et porteront ainsi l'espoir de mieux discerner les enjeux des temps futurs.

Entrée libre sur réservation (selon les places disponibles)

Pour toute information, contacter le secrétariat scientifique du Comité pour l'histoire de l'Inserm : celine.paillette@ext.inserm.fr 